\title{
Expression of functional postjunctional $\alpha_{2}$-adrenoceptors in rabbit isolated distal saphenous artery-a permissive role for angiotensin II?
}

\author{
W.R. Dunn, J.C. McGrath \& V.G. Wilson \\ Autonomic Physiology Unit, Institute of Physiology, University of Glasgow, Glasgow, G12 8QQ
}

\begin{abstract}
In the rabbit isolated distal saphenous artery, the population of postjunctional adrenoceptors is of the $\alpha_{1}$ variety under normal in vitro experimental conditions, based on the potency order of selective agonists and on the effects of the antagonists prazosin and rauwolscine against responses to UK-14304. Angiotensin II (A II, $0.05 \mu \mathrm{M})$ however, without affecting resting baseline tension, markedly enhanced responses to UK-14304, particularly at low concentrations. This previously unseen component of the response to UK-14304 was resistant to prazosin $(0.1 \mu \mathrm{M})$ but susceptible to rauwolscine $(1 \mu \mathrm{M})$. A II would therefore appear to have a permissive role for the expression of a quiescent population of postjunctional $\alpha_{2}$-adrenoceptors in the rabbit distal saphenous artery.
\end{abstract}

Introduction The demonstration of postjunctional $\alpha_{2}$-adrenoceptors in isolated vascular smooth muscle preparations is difficult, particularly in arteries (McGrath, 1982). Recently Sulpizio \& Hieble (1987) and Furuta (1988) have demonstrated an enhancement of responses to $\alpha_{2}$-adrenoceptor agonists in isolated preparations in the presence of pharmacological stimulants. The presence of Bay K 8644 or inducing tone with prostaglandin $\mathrm{F}_{2 \alpha}$, enhanced responses to BHT-920 which were prazosin-resistant and rauwolscine-sensittve, in canine isolated saphenous artery and portal vein respectively. Furthermore, the physiological stimulant angiotensin II (A II), enhances postjunctional $\alpha_{2}$-adrenoceptor function in some venous preparations (Schumann \& Lües, 1983; Daly et al., 1988a). In this study we have examined the influence of A II on responses to the selective $\alpha_{2}$-adrenoceptor agonist UK-14304 (5bromo-6-[2-imidazolin-2-ylamino]-quinoxaline) in the rabbit distal saphenous artery.

Methods Male albino rabbits $(2.5-2.7 \mathrm{~kg})$ were killed by stunning followed by exsanguination. A length of distal saphenous artery from either leg was placed in physiological salt solution (for composi- tion: see Daly et al., 1988a) containing propranolol $(1 \mu \mathrm{M})$ and cocaine $(10 \mu \mathrm{M})$. The artery was then divided into six 'ring' segments (3-4 mm long) and prepared for recording of isometric tension.

After equilibration, each preparation was exposed to $3 \mu \mathrm{M}(-)$-noradrenaline (NA) and $10 \mathrm{~min}$ later washed until complete relaxation was effected. After a further $45 \mathrm{~min}$ cumulative concentration-response curves (CCRC) were obtained to either NA, phenylephrine (PE) or UK-14304. In experiments involving UK-14304, following complete washout, tissues were exposed to either saline, prazosin $(0.1 \mu \mathrm{M})$ or rauwolscine $(1 \mu \mathrm{M}) 45 \mathrm{~min}$ before a second CCRC. In some tissues A II $(0.05 \mu \mathrm{M})$ was added $15 \mathrm{~min}$ before UK14304. Results are expressed as the percentage (mean \pm s.e.mean) of the maximum response to NA or as the percentage of the maximum response of the first CCRC to UK-14304. Differences between means were considered statistically significant if $P<0.05$ : Student's $t$ test (paired or unpaired).

Results All three agonists produced concentrationdependent contractions in the distal saphenous artery. Both NA and PE were full agonists while UK-14304 was a partial agonist producing approximately $65 \%$ of the NA maximum response. The relative potency of the three agonists was, NA > PE > UK-14304 (Figure 1a). The responses to UK-14304 were antagonized by both $1 \mu \mathrm{M}$ rauwolscine ( 3 fold rightward displacement) and $0.1 \mu \mathrm{M}$ prazosin (100 fold parallel rightward displacement) (Figure 1b).

A II $(0.05 \mu \mathrm{M})$ produced a transient contraction (duration $10-12 \mathrm{~min}$ ) which relaxed to baseline before the CCRC to UK-14304 was started. A II produced a marked increase in sensitivity to low concentrations of UK-14304, with no increase in the maximum response, resulting in a change in the slope of the CCRC to UK-14304 (Figure 1c). The magnitude of the displacement produced by A II for the threshold concentration of UK-14304 was 


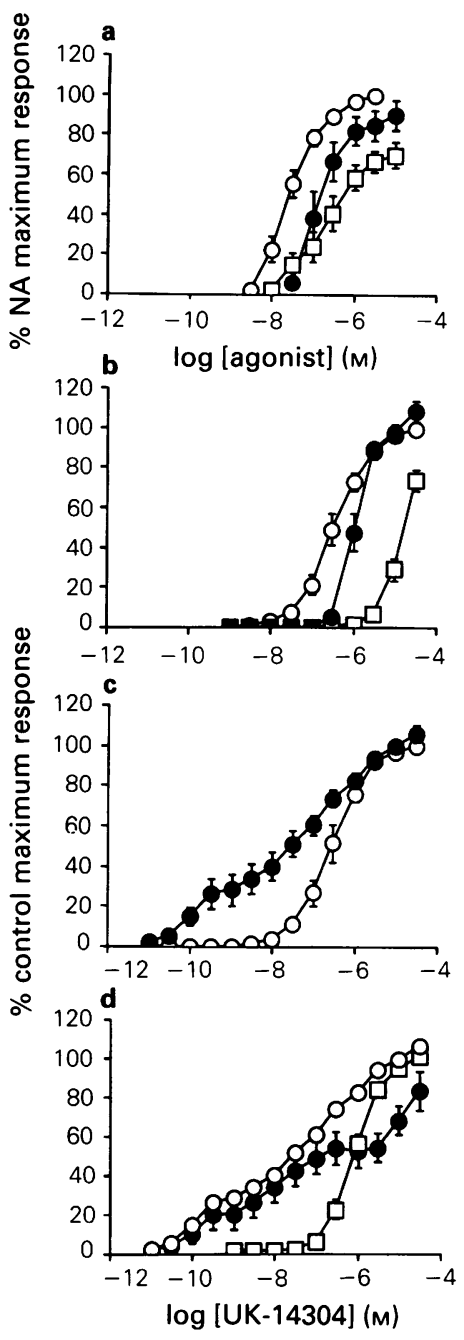

Figure 1 The effects of agonists and antagonists at $\alpha$ adrenoceptors and the influence of angiotensin II (A II) in the rabbit isolated distal saphenous artery. (a) The contractile responses to the agonists (-)-noradrenaline (NA) (O), phenylephrine (PE) (O) and UK-14304 ( $\square$ ). (b) The effect of rauwolscine $1 \mu \mathrm{M}(O)$ and prazosin $0.1 \mu \mathrm{M}(\square)$ on contractions elicited by UK-14304 (O). (c) The effect of A II $0.05 \mu \mathrm{M}(O)$ on contractions elicited by UK-14304 (O). (d) The effect of prazosin $0.1 \mu \mathrm{M}(O)$ and rauwolscine $1 \mu \mathrm{M}(\square)$ on contractions elicited to UK-14304 (O) in the presence of A II $0.05 \mu \mathrm{M}$. All responses are expressed as a percentage of either the maximum response to NA (a) or the maximum response in the control cumulative concentration-response curve to UK-14304 (b, c, d) and are the mean of 6-7 observations of different animals. The vertical lines indicate the s.e.mean. approximately 300 fold: $10^{-8} \mathrm{M}$ in the absence of A II compared to $10^{-11} \mathrm{M}$ in the presence of A II. Prazosin $(0.1 \mu \mathrm{M})$ in the presence of A II, was ineffective against the 'uncovered responses' to low concentrations of UK-14304 but displaced the upper portion of the CCRC. Rauwolscine $(1 \mu \mathrm{M})$ prevented the potentiating effect of A II on responses to UK14304 (Figure 1d).

Discussion Although pressor responses to postjunctional $\alpha_{1}$ - and $\alpha_{2}$-adrenoceptor stimulation are easily demonstrated in vivo (McGrath, 1982), responses via postjunctional $\alpha_{2}$-adrenoceptors in isolated vascular preparations have been difficult to show. This is particularly true in arterial vessels, the likely source of $\alpha_{2}$-adrenoceptor-mediated pressor responses in whole animals, while even in veins only a few clear examples have been shown (e.g. Constantine et al., 1982; Daly et al., 1988b). There is, however, some evidence that the presence of tissue stimulants can enhance the expression of $\alpha_{2}$-adrenoceptor-mediated vasoconstriction (see Introduction). We have now, for the first time in an isolated arterial preparation, shown that activation with a physiological stimulant, namely A II, reveals a quiescent population of $\alpha_{2}$-adrenoceptors in a vessel whose response was mediated entirely by $\alpha_{1}$-adrenoceptors in the absence of A II. This is associated with a marked increase in the sensitivity of the preparation (up to 300 fold) to the selective $\alpha_{2}$-adrenoceptor agonist UK-14304.

The rabbit distal saphenous artery clearly responds to $\alpha$-adrenoceptor agonists through $\alpha_{1}$-adrenoceptors under normal, in vitro, experimental conditions. Firstly, the relative potency of agonists is NA > PE > UK-14304 and secondly, prazosin and rauwolscine produced antagonism against UK14304 consistent with that shown previously at $\alpha_{1}$-adrenoceptors in other rabbit blood vessels: prazosin (estimated - $\log K_{\mathrm{b}}$ value 8.7), rauwolscine (estimated $-\log K_{b}$ value 6.3) (see Daly et al., 1988c). A II, without altering the resting baseline tension, produced a marked increase in sensitivity to UK14304. Thus, previously absent responses were 'uncovered' at low concentrations of UK-14304: these responses were unaffected by prazosin $(0.1 \mu \mathrm{M}$ : a concentration 50 times greater than its $K_{\mathrm{b}}$ value at $\alpha_{1}$-adrenoceptors in this tissue). The CCRC at higher concentrations of UK-14304 however, was shifted to the right, indicating the continued expression of responses via $\alpha_{1}$-adrenoceptors. In contrast, in the presence of A II, rauwolscine $(1 \mu \mathrm{M})$ antagonized the response to UK-14304 more effectively than prazosin $(0.1 \mu \mathrm{M})$, in effect preventing the increase in sensitivity produced by A II. This confirms that the 'uncovered' responses were mediated by postjunctional 
$\alpha_{2}$-adrenoceptors. The present study shows that a stimulating agent is required for expression of functional postjunctional $\alpha_{2}$-adrenoceptors in the rabbit distal saphenous artery. The requirement for physiological stimulants, such as AII, could therefore explain the hitherto perplexing differences in the expression of postjunctional $\alpha_{2}$-adrenoceptors between in vivo and in vitro preparations.

\section{References}

CONSTANTINE, J.W., LEBEL, W. \& ARCHER, R. (1982). Functional postsynaptic alpha $2^{-}$, but not

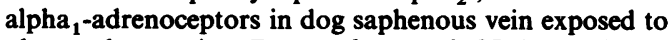
phenoxybenzamine. Eur. J. Pharmacol., 85, 325-329.

DALY, C.J., DUNN, W.R., MCGRATH, J.C. \& WILSON, V.G. (1988a). Effect of angiotensin II on responses mediated via postjunctional $\alpha_{1}$ - and $\alpha_{2}$-adrenoceptors in isolated rabbit blood vessels. Br. J. Pharmacol., 95, 699P.

DALY, C.J., DUNN, W.R., McGRATH, J.C. \& WILSON, V.G. (1988b). An attempt at selective protection from phenoxybenzamine of postjunctional $\alpha$-adrenoceptor subtypes mediating contractions to noradrenaline in the rabbit isolated saphenous vein. Br. J. Pharmacol., 95, 501-511.

DALY, C.J., MCGRATH, J.C. \& WILSON, V.G. (1988c). An examination of the postjunctional $\alpha$-adrenoceptor subtypes for (-)-noradrenaline in several isolated blood vessels from the rabbit. Br. J. Pharmacol., 95, 473-484.
This work was supported by the SERC and Roche Products UK as part of Cooperative Research Grant. Support of the Medical Research Funds of the University of Glasgow is gratefully acknowledged. We thank I.A. Dainty for helpful comments.

FURUTA, T. (1988). Precontraction-induced contractile response of isolated canine portal vein to alpha-2 adrenoceptor agonists. Naunyn-Schmiedebergs Arch. Pharmacol., 337, 525-530.

MCGRATH, J.C. (1982). Evidence for more than one type of post-junctional alpha-adrenoceptor. Biochem. Pharmacol., 31, 467-484.

SCHUMANN, H.-J. \& LUES, I. (1983). Postjunctional alphaadrenoceptors in the isolated saphenous vein of the rabbit. Naunyn-Schmiedebergs Arch. Pharmacol., 323, 328-334.

SULPIZIO, A. \& HIEBLE, J.P. (1987). Demonstration of $\alpha_{2}$-adrenoceptor-mediated contraction in the isolated canine saphenous artery treated with Bay K 8644. Eur. J. Pharmacol., 135, 107-110.

(Received September 21, 1988 Accepted October 26, 1988) 